\title{
LIDSTONE IN THE CONTINUOUS CASE
}

by

\author{
Ragnar Norberg
}

\begin{abstract}
A generalized version of the classical Lidstone theorem, which deals with the dependency of reserves on technical basis and contract terms, is proved in the case where premiums are payable continuously and insurances are payable at the noment of death.
\end{abstract}

Key words: Net level reserves, changes in technical basis, continuous payments, Thiele's differential equation.

\section{Introduction}

A useful tool for determining the effect on reserves of changes in technical basis and contract terms is Lidstone's (1905) theorem with extensions due to Baillie (1951) and Gershenson (1951). These authors treated the "discrete" case where premiums and benefits are payable at certain terms, viz. annually for premiums and annuities and at the end of the year of death for insurances. An account of this theory is offered in Jordan's (1967) textbook.

It turns out that the results which have been established for the discrete case, easily carry over to the "continuous" case where premiums and annuities are payable continuously and insurances are payable at the moment of death. Since the structure of the proof is virtually the same in the two cases - they differ only with respect to technicalities - we turn directly to the continuous case. Anticipating events, we point out that, in accordance with what is usually observed also elsewhere in life insurance mathematics, arguments and results are actually simpler in the continuous case. 
$c_{t} \leqslant 0$ for $t \in\left(0, t_{0}\right)$

and

$c_{t} \geqslant 0$ for $t \in\left(t_{0}, n\right)$,

then

$V_{t}^{\prime} \leqslant V_{t} \quad$ for $\quad t \in(0, n)$

(ii) The result in (i) remains valid if all inequalities are reversed.

(iii) The results in (i) and (ii) remain valid if all inequalities are made strict.

Proof: Upon subtracting (1) from the corresponding relation for special basis quantities and rearranging terms, we get the differential equation

$\frac{d}{d t} r_{t}-\left(\delta^{\prime}+\mu_{x+t}^{\prime}\right) r_{t}=c_{t}, \quad 0<t<n$,

with $c_{t}$ defined by (2). The assumed equality of $v_{t}$ and $v_{t}^{\prime}$ in the limit as $t \downarrow 0$ or $t \uparrow n$ yields the boundary conditions

$r_{0+}=0$ and $r_{n-}=0$.

Multiplying in (5) by $\exp \left\{-\int_{0}^{t}\left(\delta^{\prime}+\mu_{x+s}^{\prime}\right) d s\right\}$ and forming a complete differential on the left hand side, we get the equivalent equation $\frac{d}{d t}\left[r_{t} \exp \left\{-\int_{0}^{t}\left(\delta^{\prime}+\mu_{x+s}^{\prime}\right) d s\right\}\right]=c_{t} \exp \left\{-\int_{0}^{t}\left(\delta^{\prime}+\mu_{x+s}^{\prime}\right) d s\right\}, \quad 0<t<n$.

Integrating (7) from 0 to $t$ and employing the first condition in (6), we obtain the "retrospective" formula

$r_{t}=\int_{0}^{t} c_{\tau} \exp \left\{\int_{\tau}^{t}\left(\delta^{\prime}+\mu_{x+s}^{\prime}\right) d s\right\} d \tau, \quad 0 \leqslant t \leqslant n$.

Similarly, integrating from $t$ to $n$ and employing the second condition in (6), we obtain the "prospective" formula 
$r_{t}=-\int_{t}^{n} c_{\tau} \exp \left\{-\int_{t}^{\tau}\left(\delta^{\prime}+\mu_{x+s}^{\prime}\right) d s\right\} d \tau, \quad 0 \leqslant t \leqslant n$.

Now, applying (8) for $t \leqslant t_{0}$ and (9) for $t>t_{0}$, we arrive at the conclusions of the theorem. ||

Before turning to a closer study of special cases, we note that, trivially, the conditions of the theorem could be further specified. If, for instance, $c_{t}=0$ for each $t$ in some interval $\left(0, t_{1}\right)$ or $\left(t_{2}, 0\right)$, then it is readily seen from the proof that $r_{t}=0$ in this interval. If, in particular, $c_{t}=0$ for all $t \in(0, n)$, then $r_{t}=0$ for all $t$. The possibility of $t_{0}$ being 0 or $\mathrm{n}$ is not treated in the theorem since it would imply the case just mentioned, with vanishing $c_{t}$.

\section{Applications of the basic theorem}

We immediately obtain "continuous" analogies of the two corollaries presented in Jordan (1967) for the discrete case:

\section{Corollary 1 . Assume that premiums are payable continuously} at a constant rate throughout the duration of the policy. Then, provided that the standard reserve increases with duration, we have:

(i) An increase in the force of interest produces a decrease in reserves.

(ii) A decrease in the force of interest produces an increase in reserves.

Proof: Under the assumptions of the corollary we have $\mu_{x+t}^{\prime}=$ $\mu_{x+t}$, constant premiums $\pi_{t}=\pi$ and $\pi_{t}^{\prime}=\pi^{\prime}$, say, and $h_{t}^{\prime}=h_{t}$, which makes (2) assume the form

$c_{t}=\pi^{\prime}-\pi+\left(\delta^{\prime}-\delta\right) v_{t} \cdot$ 
As $V_{t}$ is assumed to be increasing, $c_{t}$ is increasing or decreasing according as $\delta^{\prime}>\delta$ or $\delta^{\prime}<\delta$. Thus items (i) and (ii) in the corollary follow from the corresponding items in the theorem. ||

As noted by Jordan, the requirement that $V_{t}$ be increasing is normally satisfied in the case of pure endowment and whole lifeor endowment insurance, but not in the case of term insurance.

The corollary in Jordan that corresponds to our Corollary 1 studies, within the discrete set-up, the effect of a change in the annual interest rate, which is equivalent to the change in the force of interest considered here. The practical relevance of studying the effects of such changes is obvious.

The second of Jordan's corollaries deals with changes in the annual rates of mortality, $q_{x}=1-\exp \left\{-\int_{0}^{1} \mu_{x+s} d s\right\}, \quad x=0,1, \ldots, \omega-1$.

Results are obtained for uniform changes in the mortality rate, that is, $q_{x}^{\prime}=q_{x}+k$ for all $x=0,1, \ldots, w-1$. Such changes are rarely, if ever, encountered in practice, and the value of the mentioned corollary therefore is mainly due to the indication it gives of what can be expected by more realistic variations in mortality assurmptions. The continuous set-up, however, allows for results of immediate practical significance since the critical function in this case depends on the mortality laws through the forces of mortality instead of the annual mortality rates. We first make some definitions:

Starting from a standard $\mu_{y}$, we shall say that the special $\mu_{y}^{\prime}$ represents a progressive increase (decrease) in mortality if $\mu_{y}^{\prime} \geqslant \mu_{y} \quad\left(\mu_{y}^{\prime} \leqslant \mu_{y}\right)$ for all $y$ and $\left|\mu_{y}^{\prime}-\mu_{y}\right|$ is a non-vanishing and non-decreasing function of $y$. A degressive increase (decrease) 
in mortality is defined by replacing "non-decreasing" by "nonincreasing" in the above definition. A change in mortality which is at the same time both progressive and degressive, that is, $\mu_{y}^{\prime}=$ $\mu_{y}+k$ for all $y$, will be called uniform. These notions of change in mortality comprise any changes in one of the three parameters of the Gompertz-Makeham intensity $\mu_{y}=\alpha+\beta c^{y}$; a change in $\alpha$ is uniform, and any change either in $\beta$ or in $c$ is progressive. We shall dernonstrate the following results:

Corollary 2. Consider an n-year endowment life insurance with sum $\mathbf{s}$ and premiums payable continuously at a constant rate throughout the duration of the policy. If the standard reserve increases with duration, then we have:

(i) A uniform or degressive increase in mortality produces a decrease in reserves.

(ii) A uniform or degressive decrease in mortality produces an increase in reserves.

Proof: We now have $\delta^{\prime}=\delta, \pi_{t}=\pi$ and $\pi_{t}^{\prime}=\pi$ (constants), $h_{t}=s \mu_{x+t}$, and $h_{t}^{\prime}=s \mu_{x+t}^{\prime}$. Substituting this in (2), we get $c_{t}=\pi^{\prime}-\pi+\left(\mu_{x+t}-\mu_{x+t}^{\prime}\right)\left(s-v_{t}\right)$.

The conclusions of the corollary result from the basic theorem by noting that $s-V_{t}$ is non-increasing and non-negative. ||

A number of further special results may be derived from Lidstone's theorem. Corollary 2 is easily modified so as to yield a result for an n-year pure endowment with constant, continuous premium throughout the duration of the policy. In this case a change in mortality gives

$c_{t}=\pi^{\prime}-\pi+\left(\mu_{x+t}^{\prime}-\mu_{x+t}\right) V_{t}$, 
and it can be concluded that a uniform or progressive increase in ). mortality produces a decrease in reserves, provided that the standard reserve increases with duration (as will normally be the case We close our present discussion with an interesting application that has been outlined within the discrete framework by Sverdrup (1982). Consider a standard basis with $h_{n}=\pi_{0}=0$ and constant premium intensity $\pi_{t}=\pi, 0<t<n$. Let the special basis differ from the standard one only by a change to natural premium payment, that is $\pi_{0}^{\prime}=0$ and $\pi_{t}^{\prime}=h_{t}$ for $0<t<n$. Then (1) applied to $\mathrm{V}_{t^{\prime}}$, with the initial condition $\mathrm{V}_{\mathrm{O}_{+}}^{\prime}=0$, implies that $v_{t}^{\prime}=0$ for all $t$, and so the hasic theorem above provides a criterion for deciding whether the standard reserve satisfies the requirement of being non-negative for all t. The critical function in (2) now reduces to

$c_{t}=h_{t}-\pi$

and we conclude from part (i) of the main theorem that a sufficient condition for $v_{t}$ to be non-negative is that $h_{t}$ is a nondecreasing function of $t$. On the other hand, if $h_{t}$ is strictly decreasing, as it may be for instance for decreasing life insurances, we can conclude that $v_{t}$ is negative in $(0, n)$.

\section{References}

Baillie, D.C. (1951): The equation of equilibrium. Trans. Soc. of Actuaries $3,74-81$.

Gershenson, H. (1951): Reserves by different mortality tables. Trans. Soc. of Actuaries 3, 68-73.

Jordan, C.W. (1967): Life Contingencies, 2nd ed. Soc. of Actuaries, Chicago.

Lidstone, G.J. (1905): Changes in pure premium values consequent upon variations in the rate of interest or rate of mortality. Journ. Institute of Actuaries 3 .

Sverdrup, E. (1982): Forsikringsfondet i livsforsikring. Statistical memoirs No. 1, 1982. Institute of Mathematics, University of Oslo. (In Norwegian.) 